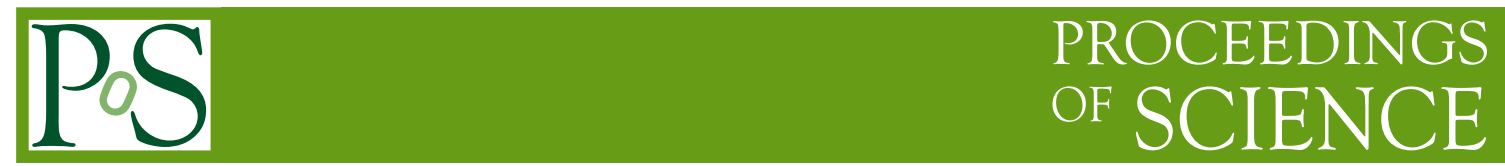

\title{
SMEFT, a theory for SM deviations
}

\section{Giampiero Passarino*t}

Dipartimento di Fisica Teorica, Università di Torino, INFN, Sezione di Torino, Italy

E-mail: giampiero@to.infn.it

During Run 1 of LHC Standard Model deviations have been analyzed in the context of the socalled kappa framework. Perspectives on a new framework for improving the experimental strategy are briefly discussed.

38th International Conference on High Energy Physics

3-10 August 2016

Chicago, USA

\footnotetext{
* Speaker.

${ }^{\dagger}$ Work supported by the Research Executive Agency (REA) of the European Union under the Grant Agreement PITN-GA-2012-316704 (HiggsTools).
} 


\section{Introduction}

Before the 2012 discovery the hypothesis was the Standard Model (SM) and $m_{\mathrm{H}}$ the unknown, therefore bounds on $m_{\mathrm{H}}$ were derived through a comparison with high-precision data. At LHC, after the discovery, the unknowns are SM deviations, given that the SM is fully specified and deviations are constrainable. Of course, the definition of SM deviations requires a characterization of the underlying dynamics. Notice that, so far, all the available studies on the couplings of the new resonance conclude it to be compatible with the Higgs boson of the SM within present precision, and, as of yet, there is no direct evidence for new physics phenomena beyond the SM.

\section{SM deviations}

Best fit results for the production signal strengths for the combination of ATLAS and CMS data are given in Ref. [1]. The next step will be to identify the optimal framework for SM deviations, i.e. a theory replaces a framework after testing confirms the hypothesis.

kappa framework A procedure used at LO, partially accommodating factorizable QCD corrections, introduced in Ref. [2]. In general, there are contributions which induce sizeable corrections unrelated to the SM ones [3] but not electroweak (EW) corrections, to parametrize SM deviations. It amounts to replace $\mathscr{L}_{\mathrm{SM}}(\{m\},\{g\})$ with $\mathscr{L}\left(\{m\},\left\{\kappa_{g} g\right\}\right)$, where $\{m\}$ denotes the SM masses, $\{g\}$ the SM couplings and $\kappa_{g}$ are the scaling parameters. This is the framework used during Run 1.

EFT/SMEFT Theories where we expand observables perturbatively in a small coupling constant and in a ratio of scales can be regarded as examples of effective field theory (EFT), e.g. SM effective field theory (SMEFT):

$$
\mathscr{L}=\mathscr{L}_{\mathrm{SM}}+\sum_{n>4} \sum_{i=1}^{N_{n}} \frac{a_{i}^{n}}{\Lambda^{n-4}} \mathscr{O}_{i}^{(d=n)},
$$

with arbitrary Wilson coefficients $a_{i}^{n}$ which, however, give the leading amplitudes in an exactly unitary $\mathrm{S}$-matrix at energies far below the scale of new physics, $\Lambda$. The theory is (strictly) nonrenormalizable, which means that an infinite number of higher operators must be included. Nevertheless there is a consistent expansion of amplitudes in power of $\mathrm{v} / \Lambda, E / \Lambda$, where $\mathrm{v}$ is the Higgs VEV and $E$ is the typical scale at which we measure the process.

Phenomenological Lagrangians Theory deals with the well founded theoretical results obtained from first principles, while phenomenology deals with not so well founded effective models with a smaller domain of application [4]. Any phenomenological approach, e.g. an extension of the SM Lagrangian with a limited number of interactions (like HVV and Hfff), is a reasonable starting point to describe limits on SM deviations, see Refs. [5, 6, 7]. While this outcome is much less desirable than dealing with a consistent SMEFT it is important to recognize that the difference relates to possibility of including theory uncertainties. 
Pseudo-Observables Pseudo observables (POs) are a platform between realistic observables and theory parameters, allowing experimentalists and theorists to meet half way between, without theorists having to run full simulation and reconstruction and experimentalists fully unfolding to modeldependent parameter spaces $[8,9]$. In other words, POs answer the question "how to measure in order to preserve the data for a long time?"

\section{SMEFT}

The rationale for constructing SMEFT, i.e. $S^{\text {eff }}(\Lambda), \forall \Lambda<\infty$, has been described in Ref. [10]; the main assumption is that there is no fundamental scale above which $S^{\operatorname{eff}(\Lambda)}$ is not defined [11]. Of course, $S^{\text {eff }}(\Lambda)$ loses its predictive power if a process at $E=\Lambda$ requires an infinite number of renormalized parameters [12]. This general approach is better than a more (a priori) restrictive framework (e.g. NLO SMEFT corrections should be included only in particular "well-defined" [sic] situations) since the former covers more ground and relies on fewer and/or weaker assumptions. The basis for NLO calculus of the SMEFT theory has been developed in Ref. [13] and in Refs. [14, 15], see also Ref. [16], in this original, unedited version. The issue of theoretical uncertainty associated with missing higher orders (MHOU) has been raised and discussed in Ref. [17]. The interplay between SMEFT and POs has been introduced in Ref. [8]. To explain SMEFT in a nutshell consider a process described by some SM amplitude, $\mathscr{A}_{\mathrm{SM}}=\sum_{i=1, n} \mathscr{A}_{\mathrm{SM}}^{(i)}$, where $i$ labels gauge-invariant sub-amplitudes. In the extension the same process is given by a contact term or a collection of contact terms of $\operatorname{dim}=6$; for instance, direct coupling of $\mathrm{H}$ to $\mathrm{VV}(\mathrm{V}=\gamma, \mathrm{Z}, \mathrm{W})$. In order to construct the theory one has to select a set of $\operatorname{dim}=6$ operators and to start the complete procedure of renormalization [13]. Going from SM to SMEFT we modify the amplitude as follows:

$$
\mathscr{A}_{\mathrm{SMEFT}}^{\mathrm{LO}}=\sum_{i=1, n} \mathscr{A}_{\mathrm{SM}}^{(i)}+i g_{6} \kappa_{\mathrm{c}}, \quad \mathscr{A}_{\mathrm{SMEFT}}^{\mathrm{NLO}}=\sum_{i=1, n} \kappa_{i} \mathscr{A}_{\mathrm{SM}}^{(i)}+i g_{6} \kappa_{\mathrm{c}}+g_{6} \sum_{i=1, \mathrm{~N}} a_{i} \mathscr{A}_{\mathrm{nfc}}^{(i)},
$$

where $g_{6}^{-1}=\sqrt{2} G_{\mathrm{F}} \Lambda^{2}$. The last term collects all loop contributions that do not factorize and the coefficients $a_{i}$ are Wilson coefficients. The $\kappa_{i}$ are linear combinations of the $a_{i}$. Contributions from interference effects between the different (gauge invariant) sub-amplitudes of Eq.(3.1) provide some sensitivity to the relative signs of the Higgs boson couplings to different particles. Therefore, additional coupling modifiers are introduced in a consistent way, i.e. without making the assumption of higher orders factorization. The interplay between integrating out heavy scalars and the SM decoupling limit has been discussed in Refs. $[18,19,20]$. In the very general case the SM decoupling limit cannot be obtained by making only assumptions about one parameter.

We conclude that SMEFT gives the correct generalization of the original $\kappa$-framework at the price of introducing additional, non-factorizable, terms in the amplitude (i.e. more resolved coupling modifiers). Furthermore, the main message from Run 1 is: it is important to check the apparent minimality of the Higgs sector as it is important to anticipate deviations. Of course, not only is there LHC, there are electroweak precision data (EWPD). Measurements of the W mass provide an important consistency check of the SM and constrain BSM physics. Global constraints of the SMEFT have been developed in Ref. [17], with results that show how the SMEFT theory error should not be neglected in future fit errors. Preliminary results of a Bayesian fit to the Wilson coefficients using data on EW precision observables and Higgs boson signal strengths have been presented in Ref. [21]. 


\section{First results in SMEFT}

The work of Refs. $[22,22]$ has shown that the extra error introduced in these measurements due to SMEFT higher dimensional operators is subdominant to the current experimental systematic errors. This means that the leading challenge to interpreting these measurements in the SMEFT is the pure theoretical uncertainty in how these measurements are mapped to Lagrangian parameters. There is now a tendency to agree with having a "truncation" error in SMEFT, and the recommendation is to quote it separately. Referring to the case in which we observe some deviation from the SM in the future, if the experimental precision allows, we will be able to test NLO corrections and/or dim $=8$ effects. If on the other hand, no deviation is observed and limits are set through a LO procedure, then NLO effects will be part of the MHOU.

Inclusion of EWPD in a global fit deserves additional comments. Usually bounds on the coefficients are obtained in two ways: individual coefficients are switched one one at the time, or marginalized in a simultaneous fit. The global constraint picture on SMEFT parameters has been updated with the conclusion that stronger constraints can be obtained by using some combinations of Wilson coefficients, when making assumptions on the UV completion of the SM. Furthermore, strong bounds at the per-mille or sub-per-mille level on some combinations of Wilson coefficients in the Effective Lagrangian can be artificially enhanced in fits of this form in detail. As discussed in Refs. [23, 24] a few select kinematic distributions can be used to collect information on modified Higgs couplings, for example in the gluon fusion production process. In the top-gluon-Higgs sector one can compare three different analysis strategies: a) a modified $p_{\mathrm{T}}$ spectrum of boosted Higgs production in gluon fusion, b) off-shell Higgs production [25, 26] i.e. pole observables vs. tail of distributions, and c) a measurement of the gluon fusion vs $\overline{\mathrm{t}} \mathrm{tH}$ production rates. Unfortunately, explicit threshold effects in boosted Higgs production are too small to be observable in the near future [27]. Global analyses including kinematic information in all Higgs channels cannot rely on the kappa framework, but they can be based on SMEFT. Such analyses provide potentialities and challenges at the same time. Ref. [28] deals with Higgs production through weak boson fusion with subsequent decay to bottom quarks. By combining jet substructure techniques and matrix element methods in different limits the authors motivate this channel as a probe of the bottom-Yukawa interactions in the boosted regime. The possibility to separate in gluon fusion loop-induced Higgs boson production from point-like production has been examined in Ref. [29].

Several (theoretical) analyses have been performed with the available Run 1 data, as summarized in Refs. [30, 28, 31, 32]. These analyses always use a subset of the full Warsaw basis and show a good agreement, with differences due to different sets of assumptions, showing good agreement with the zero SM deviation hypothesis. To be more precise, the best precisions achieved are approximately $30 \%$ for the ratio of cross sections $\mathrm{VBF} / \mathrm{ggF}$ (vector boson fusion and gluon-gluon fusion) and for the ratios of branching fractions, $\mathrm{BR}(\mathrm{WW}) / \mathrm{BR}(\mathrm{ZZ})$ and $\mathrm{BR}(\gamma \gamma) / \mathrm{BR}(\mathrm{ZZ})$. The ratios of coupling modifiers (kappa parameters) are measured with precisions of approximately $10 \rightarrow 20 \%$. What has been learnt is that kinematic distributions can significantly improve the multi-dimensional parameter by resolving strong correlations induced by total rate measurements. 


\section{Conclusions}

After the discovery we have a conventional vision: some very different physics occurs at Planck scale, SM is just an effective field theory. What about the next SM? A new weakly coupled renormalizable model? A tower of EFTs? The SMEFT framework is useful because one can set limits on the effective coefficients in a model-independent way. This is why SMEFT in the bottomup approach is so useful: we do not know what the tower of UV completions is (or if it exists at all) but we can formulate the SMEFT and perform calculations with it without needing to know what happens at arbitrarily high scales. On the other hand interpreting such limits as bounds on UV models does require some assumption of the UV dynamics [33]. A different vision? Is the SM close to a fundamental theory?

\section{References}

[1] ATLAS, CMS collaboration, G. Aad et al., Measurements of the Higgs boson production and decay rates and constraints on its couplings from a combined ATLAS and CMS analysis of the LHC pp collision data at $\sqrt{s}=7$ and $8 \mathrm{TeV}$, JHEP 08 (2016) 045, [1606.02266].

[2] LhC Higgs Cross Section Working Group collaboration, A. David, A. Denner, M. Duehrssen, M. Grazzini, C. Grojean, G. Passarino et al., LHC HXSWG interim recommendations to explore the coupling structure of a Higgs-like particle, 1209.0040.

[3] R. Gauld, B. D. Pecjak and D. J. Scott, $Q C D$ radiative corrections for $h \rightarrow b \bar{b}$ in the Standard Model Dimension-6 EFT, 1607.06354.

[4] S. Hartmann, "Effective field theories, reduction and scientific explanation." Studies in History and Philosophy of Modern Physics 32B, 267-304, 2001.

[5] R. S. Gupta, A. Pomarol and F. Riva, BSM Primary Effects, Phys. Rev. D91 (2015) 035001, [1405.0181].

[6] F. Riva, BSM primaries: the physics of the SM effective field theory, Nuovo Cim. C38 (2016) 149.

[7] A. Falkowski, B. Fuks, K. Mawatari, K. Mimasu, F. Riva and V. sanz, Rosetta: an operator basis translator for Standard Model effective field theory, Eur. Phys. J. C75 (2015) 583, [1508 . 05895].

[8] A. David and G. Passarino, Through precision straits to next standard model heights, Rev. Phys. 1 (2016) 13-28, [1510.00414].

[9] G. Isidori, A. Greljo, J. Lindert, D. Marzocca and G. Passarino, "Pseudo-observables in Higgs physics.” LHCHXSWG-DRAFT-INT-2016-004, https://cds.cern.ch/record/2138023, 2016.

[10] G. Passarino, NLO Standard model effective field theory for Higgs and EW precision data, in 13th DESY Workshop on Elementary Particle Physics: Loops and Legs in Quantum Field Theory (LL2016) Leipzig, Germany, April 24-29, 2016, 2016. 1607.01236.

[11] K. Costello, "Renormalization and Effective Field Theory." Mathematical Surveys and Monographs Volume 170, American Mathematical Society, 2011.

[12] J. Preskill, Gauge anomalies in an effective field theory, Annals Phys. 210 (1991) 323-379.

[13] M. Ghezzi, R. Gomez-Ambrosio, G. Passarino and S. Uccirati, NLO Higgs effective field theory and kappa-framework, JHEP 07 (2015) 175, [1505.03706]. 
[14] C. Hartmann and M. Trott, On one-loop corrections in the standard model effective field theory; the $\Gamma(\mathrm{H} \rightarrow \gamma \gamma)$ case, JHEP 07 (2015) 151, [1505.02646].

[15] C. Hartmann and M. Trott, Higgs Decay to Two Photons at One Loop in the Standard Model Effective Field Theory, Phys. Rev. Lett. 115 (2015) 191801, [1507.03568].

[16] G. Passarino and M. Trott, "The Standard Model Effective Field Theory and Next to Leading Order." LHCHXSWG-DRAFT-INT-2016-005, https://cds.cern.ch/record/2138031, 2016.

[17] L. Berthier and M. Trott, Towards consistent Electroweak Precision Data constraints in the SMEFT, JHEP 05 (2015) 024, [1502 . 02570].

[18] F. del Aguila, Z. Kunszt and J. Santiago, One-loop effective lagrangians after matching, 1602.00126 .

[19] M. Boggia, R. Gomez-Ambrosio and G. Passarino, Low energy behaviour of standard model extensions, JHEP 05 (2016) 162, [1603 . 03660].

[20] B. Henning, X. Lu and H. Murayama, How to use the Standard Model effective field theory, JHEP 01 (2016) 023, [1412.1837].

[21] J. de Blas, M. Ciuchini, E. Franco, S. Mishima, M. Pierini, L. Reina et al., Electroweak precision observables and Higgs-boson signal strengths in the Standard Model and beyond: present and future, 1608.01509 .

[22] L. Berthier, M. Bjørn and M. Trott, Incorporating doubly resonant $W^{ \pm}$data in a global fit of SMEFT parameters to lift flat directions, 1606.06693.

[23] J. Brehmer, A. Freitas, D. Lopez-Val and T. Plehn, Pushing Higgs Effective Theory to its Limits, Phys. Rev. D93 (2016) 075014, [1510 . 03443$].$

[24] A. Biekötter, J. Brehmer and T. Plehn, Pushing Higgs Effective Theory over the Edge, 1602 . 05202.

[25] M. Ghezzi, G. Passarino and S. Uccirati, Bounding the Higgs Width Using Effective Field Theory, PoS LL2014 (2014) 072, [1405 . 1925].

[26] C. Englert, M. McCullough and M. Spannowsky, Combining LEP and LHC to bound the Higgs Width, Nucl. Phys. B902 (2016) 440-457, [1504 . 024 58].

[27] M. Buschmann and F. Yu, Angular observables for spin discrimination in boosted diboson final states, 1604.06096.

[28] C. Englert, O. Mattelaer and M. Spannowsky, Measuring the Higgs-bottom coupling in weak boson fusion, Phys. Lett. B756 (2016) 103-108, [1512 . 03429].

[29] U. Langenegger, M. Spira and I. Strebel, Testing the Higgs Boson Coupling to Gluons, 1507. 01373.

[30] C. Englert, R. Kogler, H. Schulz and M. Spannowsky, Higgs coupling measurements at the LHC, Eur. Phys. J. C76 (2016) 393, [1511.05170].

[31] J. Ellis, V. Sanz and T. You, The Effective Standard Model after LHC Run I, JHEP 03 (2015) 157, [1410.7703].

[32] O. Bessidskaia Bylund, F. Maltoni, I. Tsinikos, E. Vryonidou and C. Zhang, Probing top quark neutral couplings in the Standard Model Effective Field Theory at NLO in QCD, JHEP 05 (2016) 052, [1601.08193].

[33] R. Contino, A. Falkowski, F. Goertz, C. Grojean and F. Riva, On the Validity of the Effective Field Theory Approach to SM Precision Tests, JHEP 07 (2016) 144, [1604. 06444 ]. 